\title{
THE 1-, 2-, AND 3-CHARACTERS DETERMINE A GROUP
}

\author{
H.-J. HOEHNKE AND K. W. JOHNSON
}

\begin{abstract}
A set of invariants for a finite group is described. These arise naturally from Frobenius' early work on the group determinant and provide an answer to a question of Brauer. Whereas it is well known that the ordinary character table of a group does not determine the group uniquely, it is a consequence of the results presented here that a group is determined uniquely by its "3-character" table.
\end{abstract}

\section{INTRODUCTION}

Given a finite group $G$ of order $n$ its group matrix $X_{G}$ is defined as follows. Let $x_{e}, x_{g_{2}}, \ldots, x_{g_{n}}$ be variables indexed by the elements of $G$. The $n \times n$ matrix $X_{G}$ is defined to be the matrix whose $(i, j)$ th entry is $x_{g_{i} g_{j}^{-1}}$. The group determinant $\boldsymbol{\Theta}_{G}$ of $G$ is $\operatorname{det}\left(X_{G}\right)$.

Although the group determinant first appeared in Frobenius' 1896 paper [Fr1] in which he introduced characters for arbitrary groups, the roots of the work go back to the discussions of Gauss on the composition of equivalence classes of quadratic forms. There is a good discussion of the background to Frobenius' work in [Ha1, Ha2]. A natural question arises as to whether nonisomorphic groups necessarily have distinct group determinants. This was posed by one of the authors in 1986 and was answered by Formanek and Sibley [FS] in 1990, the positive answer being somewhat surprising. In fact there were already two early remarks in the literature apart from the work of Gauss quoted above that pointed in this direction. Both of these were described in apostrophies as strange ("merkwürdig") by their authors and were disregarded for a long time within the development of algebra. The first was by Frobenius in [Fr2] on matrix transformations and the second occurred in [B] on the subject of maximal orders of quaternion algebras. Today these phenomena are subsumed under the thesis "from norm invariance to constructive structure theory and (noncommutative) arithmetics."

A further look at Frobenius' early work reveals functions $\chi^{(k)}: G^{k} \rightarrow \mathbb{C}$ defined below in the case where $k \leq 3$ that appeared in his algorithm to calculate the factor of a group determinant that corresponds to an irreducible character $\chi$. Some of the properties of these " $k$-characters" have been explored in [J] where it is shown that the $k$-characters corresponding to distinct irreducible characters $\chi_{i}, \chi_{j}$ are orthogonal in the sense that

$$
\sum_{\underline{g} \in G^{k}} \chi_{i}^{(k)}(\underline{g}) \bar{\chi}_{j}^{(k)}(\underline{g})=0 .
$$

Received by the editors March 20, 1991 and, in revised form, February 20, 1992.

1991 Mathematics Subject Classification. Primary 15A15, 16A26, 16H05, 16K20, 16S99, 20B05, 20B40, 20C15. 
There is also given in $[\mathrm{J}]$ a 2-character table of a group $G$ that consists of the 2-characters corresponding to the irreducible representations of degrees greater than 1 together with "degenerate" characters.

One of the questions raised by Brauer in $[\mathrm{Br}]$ is that of determining what extra information may be added to the (ordinary) character table to completely determine a group. As a consequence of Frobenius' work and the FormanekSibley result, the knowledge of the $k$-characters $\chi^{(k)}$ of a group for all $k$ and all irreducible characters $\chi$ is sufficient to determine a group. Note that if $k>\operatorname{deg}(\chi)$ then $\chi^{(k)}=0$. The $k$-characters thus provide an answer to Brauer's question, but since the amount of work involved in calculating the $k$-characters for large $k$ is prohibitively large, it becomes interesting to examine the question of the extent to which the knowledge of the $k$-characters for small values of $k$ determine a group.

\section{3-CHARACTERS}

The authors would like to announce the following result.

Theorem. Let $G$ be a finite group, with irreducible characters $\chi_{1}, \ldots, \chi_{r}$ over an algebraically closed field $K$ with $\operatorname{char} K \neq 2$ and char $K \nmid|G|$. Then $G$ is determined up to isomorphism by the $\chi_{j}^{(k)}, j=1, \ldots, r, k=1,2,3$.

Explicit definitions of the 1-, 2-, and 3-characters corresponding to a character $\chi$ are as follows:

$$
\begin{gathered}
\chi^{1}(g)=\chi(g), \quad \chi^{2}(g, h)=\chi(g) \chi(h)-\chi(g h), \\
\chi^{3}(g, h, m)=\chi(g) \chi(h) \chi(m)-\chi(g) \chi(h m)-\chi(h) \chi(g m) \\
-\chi(m) \chi(g h)+\chi(g h m)+\chi(g m h) .
\end{gathered}
$$

The proof of the theorem depends on results in [Ho1, Ho2]; the following is a brief outline:

(i) To each irreducible character $\chi$ of a finite group $G$ there is associated a factor $\varphi_{\chi}$ of $\boldsymbol{\Theta}_{G}$.

(ii) Any factor $\varphi$ of $\boldsymbol{\Theta}_{G}$ is a norm-type form. In particular, $\varphi(x y)=$ $\varphi(x) \varphi(y)$ where $x$ and $y$ are generic elements of the group algebra of $G$.

(iii) Let $A$ be a finite-dimensional algebra over the field $K$, and let $\left\{\omega_{1}, \ldots\right.$, $\left.\omega_{n}\right\}$ be a basis. Define the structure constants $\left\{w_{i j}^{k}\right\}$ by

$$
\omega_{i} \omega_{j}=\sum w_{i j}^{k} \omega_{k}
$$

Let $N$ be a norm-type form on $A$, with

$$
N(\lambda-x)=\lambda^{m}-s_{1}(x) \lambda^{m-1}+\cdots+(-1)^{m} s_{m}(x)
$$

where $\lambda$ is an indeterminate and $x$ is a generic element,

$$
x=x_{1} \omega_{1}+\cdots+x_{n} \omega_{n} .
$$

If the discriminant of $N$ is nonzero (which is true for example when $N$ is the group determinant) it follows that from the knowledge of $s_{1}(x), s_{2}(x)$, and $s_{3}(x)$ the "symmetrised" structure constants

$$
w_{(i j)}^{k}=w_{i j}^{k}+w_{j i}^{k}
$$

can be determined. 
(iv) By [Bo, $\S 4$, Exercise 26] if $f: G \rightarrow H$ is a bijection between finite groups such that $f(g h)$ is either $f(g) f(h)$ or $f(h) f(g)$, then $f$ is either an isomorphism or an anti-isomorphism, and hence $G$ and $H$ are isomorphic.

(v) It follows from (iii) and (iv) that the 3-character of the regular representation (which is essentially the same as $s_{3}(x)$ above when $N=\Theta_{G}$ ) determines $G$.

(vi) The 3-character of the regular representation of $G$ can be formed from the 1-, 2-, and 3-characters associated to the irreducible representations of $G$.

This provides, in some sense, a more satisfactory set of invariants for a finite group. It has also been possible to give a constructive proof of the FormanekSibley theorem using the ideas in the proof of the above theorem.

Another set of invariants for finite groups has been given by Roitman in [Ro]. These invariants consist of an apparently infinite set of integers defined for pairs of integers $n, k$ that are calculated as the coefficient of the identity in the $k$ th powers of certain elements of $\mathbb{Z} G \otimes \cdots \otimes \mathbb{Z} G$ ( $n$ factors), identifying this ring with $\mathbb{Z} G^{n}$. We refer the reader to [Ro] for the details, which are of a technical nature. The 1-, 2-, and 3-characters associated to the irreducible representations of the group seem to be much more accessible, and moreover, a 3-character table can be constructed that has convenient orthogonality properties. The question of whether the 1- and 2-characters alone determine a group is addressed in [JS] where it is shown that there exist nonisomorphic pairs of groups with the same 2-character table, i.e., with the same 1- and 2-characters.

\section{REFERENCES}

[B] H. Brandt, Idealtheorie in Quaternionenalgebren, Math. Ann. 99 (1928), 1-29.

[Bo] N. Bourbaki, Algèbre. I, Paris, 1970.

[Br] R. Brauer, Representations of finite groups, Lectures in Modern Mathematics (T. L. Saaty, ed.), Wiley, 1963, pp. 133-175.

[FS] E. Formanek and D. Sibley, The group determinant determines the group, Proc. Amer. Math. Soc. 112 (1991), 649-656.

[Fr1] G. Frobenius, Über die Primfaktoren der Gruppendeterminante, S'ber. Akad. Wiss. Berlin (1896), 1343-1382.

[Fr2] _ Über die Darstellung der endlichen Gruppen durch lineare Substitutionen, S'ber. Akad. Wiss. Berlin (1898), 944-1015.

[Ha1] T. Hawkins, The origins of the theory of group characters, Arch. Hist. Exact Sci. 7 (1971), 142-170.

[Ha2] New light on Frobenius' creation of the theory of group characters, Arch. Hist. Exact Sci. 12 (1974), 217-243.

[Ho1] H.-J. Hoehnke, Über komponierbare Formen und konkordante hyperkomplexe Grössen, Math. Z. 70 (1958), 1-12.

[Ho2] - Über Beziehungen zwischen Problemen von H. Brandt aus der Theorie der Algebren und den Automorphismen der Normenform, Math. Nachr. 34 (1967), 229-255.

[J] K. W. Johnson, On the group determinant, Math. Proc. Cambridge Philos. Soc. 109 (1991), 299-311.

[JS] K. W. Johnson and Surinder Sehgal, The 2-character table does not determine a group, preprint.

[Ro] M. Roitman, A complete set of invariants for finite groups, Adv. in Math. 41 (1981), 301-311.

Mendelstr. 4, 0-1100 Berlin, Germany and Brandendurgische landesuniversität, Fachbereich Mathematik, AM Neuen Palais, 0-1571 Potsdam-Sanssouci, Germany

Department of Mathematics, The Pennsylvania State University Abington, PennsylVANIA 19001

E-mail address: kwj1@ @suvm.bitnet 